\title{
Post-NICE 2008: antibiotic prophylaxis prior to dental procedures for patients with pulmonary arteriovenous malformations (PAVMs) and hereditary haemorrhagic telangiectasia
}

IN BRIEF

- Describes PAVMs, a condition for which many patients carry a card or letter advising of the importance of antibiotic prophylaxis prior to dental procedures to prevent brain abscess.

- Clarifies how such patients do not fall into the general group reviewed recently by NICE.

- Considers appropriate antibiotics.

\author{
C. Shovlin, ${ }^{1}$ K. Bamford ${ }^{2}$ and D. Wray ${ }^{3}$
}

Recently published guidance from NICE highlights that antibiotic prophylaxis is no longer required for patients with structural heart disease at risk of infective endocarditis. The American Heart Association has published similarly less interventive guidance. Individuals with pulmonary arteriovenous malformations and hereditary haemorrhagic telangiectasia are at risk of brain abscess from dental bacteraemias. In this article we explore why these patients do not fall into the groups considered by NICE and provide recommendations to reduce their risks of dental bacteraemias, including optimising dental hygiene and use of antibiotic prophylaxis prior to dental procedures.

Dental bacteria can cause endocarditis or other infections following dental bacteraemias induced by interventional dental procedures. The recently published NICE guidance on prophylaxis against infective endocarditis has been widely disseminated. ${ }^{1}$ In the UK, the Chief Dental Officer ${ }^{2}$ and Medical Defence Union ${ }^{3}$ have published specific advice for dental practitioners highlighting that antibiotic prophylaxis is no longer required for adults and children with structural heart disease at risk of infective endocarditis. Similar conclusions were reached by the American Heart Association. ${ }^{4}$

Patients with pulmonary arteriovenous malformations (PAVMs) and heredi-

\footnotetext{
${ }^{1 *} \mathrm{NHLI}$ Cardiovascular Sciences, Imperial College London/Respiratory Medicine, Imperial College Healthcare NHS Trust, London; ${ }^{2}$ Infectious Diseases and Immunity, Imperial College London/Microbiology, Imperial College Healthcare NHS Trust, London: ${ }^{3}$ Department of Oral Medicine, University of Glasgow

*Correspondence to: Dr Claire Shovlin, Respiratory Medicine, Hammersmith Hospital, Du Cane Road, London, W120NN

Email:c.shovlin@imperial.ac.uk
}

\section{Refereed Paper}

Accepted 22 August 2008

DOI: $10.1038 /$ sj.bdj.2008.978

${ }^{\circ}$ British Dental Journal 2008; 205: 531-533 tary haemorrhagic telangiectasia (HHT) are also at risk of sequelae from dental bacteraemias. PAVMs are usually silent vascular abnormalities in the pulmonary circulation that result in a right-toleft shunt (Figs 1A and B). PAVMs most commonly occur in association with HHT (Osler-Weber-Rendu syndrome). ${ }^{5}$ This relatively rare genetic condition is recognised classically by recurrent nosebleeds, characteristic mucocutaneous telangiectasia (Fig. 1C) and anaemia due to chronic haemorrhage from nasal or gastrointestinal telangiectasia (Fig. 1D). Screening programmes in asymptomatic individuals indicate that PAVMs affect almost 50\% of HHT patients. ${ }^{6}$

For patients with PAVMs and HHT, there is a link between oral bacteria and PAVM-associated brain abscess. Antibiotic prophylaxis has been recommended for them based on the endocarditis paradigm, ${ }^{7}$ with the British Society for Antimicrobial Chemotherapy and Dental Formulary Sub-Committee approving a PAVM-specific card in 2000 (Fig. 1F). The evidence for an association between oral micro-organisms and brain abscess was strengthened by a recent manuscript. ${ }^{8}$ The majority of organisms iso- lated from brain abscess aspirates were microaerophilic and anaerobic bacteria commonly and often specifically isolated in periodontal infections. In contrast to cardiac patients with infective endocarditis, a high proportion of the PAVM brain abscess group had experienced identifiable events known to be associated with bacteraemia in the weeks preceding their abscess. ${ }^{8}$

In view of the endocarditis recommendations, what should happen now for PAVM patients?

First, it is important to consider the three sets of reasons why the recommendation for antibiotic prophylaxis was withdrawn for patients at risk of endocarditis:

i) There is no evidence that prophylactic antibiotics prevent bacteraemias $^{9,10}$ and only limited evidence from experimental models that prophylactic antibiotics reduce endocarditis incidence ${ }^{10}$

ii) The intensity of bacteraemias during everyday tooth brushing may exceed those from dental procedures ${ }^{11}$

iii) There are financial implications and medical risks (particularly anaphylaxis) to treating large numbers of 
people with antibiotics when each individually is at a low risk of endocarditis.

Next, it is important to recognise that the NICE committee and AHA did not consider people with PAVMs at risk of brain abscess.

Third, it is essential to consider the differences between the two groups of patients. The risk of brain abscess for an HHT/PAVM patient is several orders of magnitude higher than that for heart patients at risk of endocarditis. Excluding individuals whose PAVMs were diagnosed because of a brain abscess, 9.05\% $(19 / 210)$ of patients in a recent series developed brain abscesses between the ages of 9 and 70, with a 500-fold relative increased risk compared to the general population between the ages of 45 and 54 years. ${ }^{8}$ Furthermore, the mechanisms are subtly different. For heart patients, structural abnormalities are thought to provide favourable conditions in which micro-organisms may adhere and multiply, prior to the rapid clearance of bacteraemias from the blood stream..$^{9,10}$ In contrast, for PAVM patients the predisposing event is likely to include a prolonged bacteraemic period, since blood passing through the right-to-left shunt provided by PAVMs (Fig. 1B) will have reduced exposure to the capillary bed reticuloendothelial cell system. In addition, HHT/PAVM patients may have increased susceptibility to the initial bacteraemias or the subsequent pathogenic steps compared to the non-HHT population in which dental bacteraemias were studied.

For these reasons we suggest that:

- Antibiotic prophylaxis should still be given to PAVM/HHT patients

- All PAVM patients and their families should pursue strategies to optimise dental hygiene (known to reduce the risk of dental bacteraemias of all causes)

- Further research on susceptibility of the HHT population to dental bacteraemias is warranted.

\section{Antibiotic considerations}

The best choice of prophylaxis for patients with PAVM/HHT should reflect the organisms that pose the greatest risk.
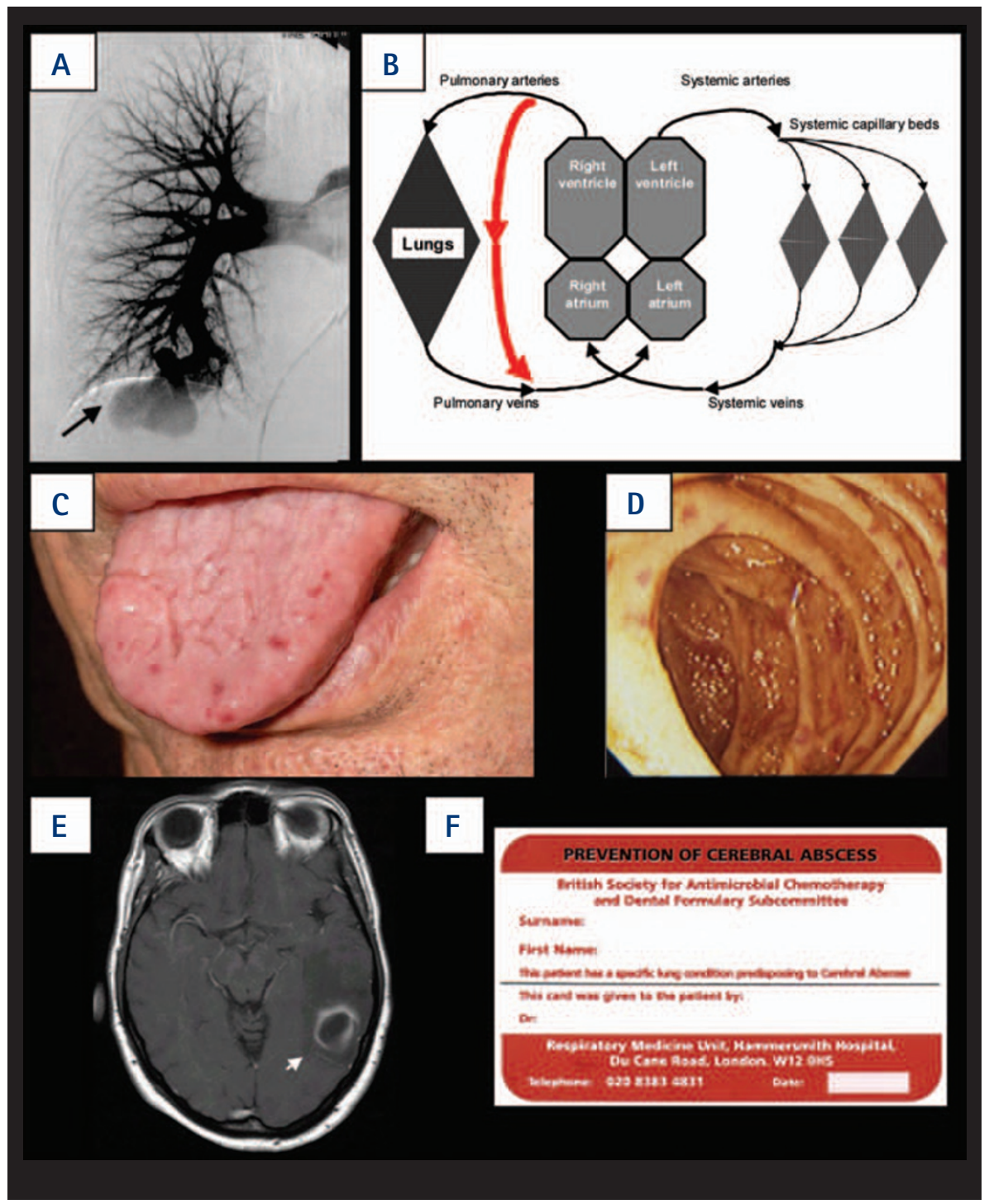

Fig. 1 PAVMs, HHT and brain abscess. A: angiographic appearance of large PAVM in the right lung (courtesy of Dr James Jackson); B: PAVMs provide a right-to-left shunt (red arrow) from pulmonary artery to pulmonary vein, bypassing the pulmonary capillary bed; C: mucocutaneous telangiectasia on the tongue and D: in the large bowel; E: contrastenhanced magnetic resonance image of cerebral abscess in a PAVM/HHT patient; F: antibiotic prophylaxis card for PAVM patients (approved 2000)

Traditionally oral bacteria and particularly the viridans group of streptococci associated with endocarditis have been predominantly sensitive to penicillin. ${ }^{12}$ This made amoxycillin a good choice. However, we are now faced with increased antimicrobial resistance and in this setting, a poly-microbial flora associated with brain abscesses. It is therefore more appropriate to consider agents with a broader spectrum. While no single agent is likely to provide comprehensive cover, a strong contender is co-amoxyclav. This would provide cover for not only the majority of the viridans streptococci but also the fastidious gram negatives and anaerobes, many of which now produce $\beta$-lactamases. ${ }^{13-16}$
In the case of individuals who are allergic to penicillin, antimicrobial susceptibility patterns suggest that clindamycin would be a good choice. Efforts should be made to ascertain the extent and nature, including antimicrobial susceptibilities, of bacteraemia associated with dental manipulations in this patient group. Until then it would appear then there is a strong case for continuing to provide targeted prophylaxis to provide the best protection for this group of patients. These issues should be discussed and debated by the HHT/PAVM clinical community.

$C L S$ and $K B B$ are grateful for support from the NIHR Biomedical Research Centre Funding Scheme. 
1. Wray D, Ruiz F, Richey R, Stokes T. Prophylaxis against infective endocarditis for dental procedures - summary of the NICE guideline. Br Dent J 2008; 204: 555-557.

2. Department of Health. NICE guidance on 'Antibiotic prophylaxis against infective endocarditis'. London: Department of Health, 2008. Chief Dental Officer letter, gateway reference 9638. http:// www.dh.gov.uk/en/Publicationsandstatistics/Lettersandcirculars/Professionalletters/Chiefdentalofficerletters/DH_083679

3. Harvey B. New guidance on antibiotic prophylaxis. Medical Defence Union website, 2 April 2008. Available at www.the-mdu.com.

4. Wilson W, Taubert K A, Gewitz M et al. Prevention of infective endocarditis: guidelines from the American Heart Association. J Am Dent Assoc 2007: 138: 739-745, 747-760.

5. Shovlin C L, Guttmacher A E, Buscarini E et al. Diagnostic criteria for hereditary haemorrhagic telangiectasia (Rendu-Osler-Weber syndrome). Am J Med Genet 2000; 91: 66-67.

6. Cottin V, Plauchu H, Bayle J-Y et al. Pulmonary arteriovenous malformations in patients with hereditary hemorrhagic telangiectasia. Am J Respir Crit Care Med 2004; 169: 994-1000

7. Chan P. Antibiotic prophylaxis for patients with hereditary hemorrhagic telangiectasia. J Am Acad Dermatol 1992; 26: 282-283.

8. Shovlin C L, Jackson J E, Bamford K B et al. Primary determinants of ischaemic stroke/brain abscess risks are independent of severity of pulmonary arteriovenous malformations in hereditary haemorrhagic telangiectasia. Thorax 2008; 63: 259-266.

9. Hall G, Hedstrom S A, Heimdahl A, Nord C E. Prophylactic administration of penicillins for endocarditis does not reduce the incidence of postextraction bacteremia. Clin Infect Dis 1993; 17: 188-194.

10. Moreillon P, Overholser C D, Malinverni R, Bille J, Glauser M P. Predictors of endocarditis in isolates from cultures of blood following dental extractions in rats with periodontal disease. J Antimicrob Chemother 1988; 20: 87-93.

11. Roberts G J. Dentists are innocent! 'Everyday' bacteremia is the real culprit: a review and assessment of the evidence that dental surgical procedures are a principal cause of bacterial endocarditis in children. Pediatr Cardiol 1999; 20: 317-325.

12. Coulter W A, Coffey A, Saunders I D, Emmerson A M. Bacteremia in children following dental extraction. J Dent Res 1990; 69: 1691-1695.

13. Wang $H \mathrm{~K}$, Chen $Y \mathrm{C}$, Teng $\mathrm{L} J$ et al. Brain abscess associated with multidrug-resistant Capnocytophaga ochracea infection. J Clin Microbio/ 2007; 45: 645-647.

14. Rega A J, Aziz S R, Ziccardi V B. Microbiology and antibiotic sensitivities of head and neck space infections of odontogenic origin. J Oral Maxillofac Surg 2006; 64: 1377-1380.

15. Kuriyama T, Williams D W, Yanagisawa M et al. Antimicrobial susceptibility of 800 anaerobic isolates from patients with dentoalveolar infection to 13 oral antibiotics. Oral Microbiol Immunol 2007; 22: 285-288.

16. Brescó-Salinas M, Costa-Riu N, Berini-Aytés $L$, Gay-Escoda C. Antibiotic susceptibility of the bacteria causing odontogenic infections. Med Oral Patol Oral Cir Bucal 2006; 11: E70-E75. 